\title{
NUEVO REGISTRO DE GOMPHOTHERIUM HONDURENSIS (FRICK, 1933) (PROBOSCIDEA, GOMPHOTERIIDAE) PARA EL MIOCENO SUPERIOR DE EL SALVADOR, AMÉRICA CENTRAL
}

\author{
A NEW RECORD OF GOMPHOTHERIUM HONDURENSIS (FRICK, 1933) \\ (PROBOSCIDEA, GOMPHOTERIIDAE) ON THE UPPER MIOCENE OF EL \\ SALVADOR, CENTRAL AMERICA
}

\author{
Daniel H. Aguilar ${ }^{1} \&$ César A. Laurito*2\&3 \\ ${ }^{1}$ Museo de Historia Natural de El Salvador; San Salvador, El Salvador \\ ${ }^{2}$ Instituto Nacional de Aprendizaje (INA) \\ ${ }^{3}$ Museo Nacional de Costa Rica, Departamento de Historia Natural. Apdo. \\ Postal 203-2200, Coronado, San José, Costa Rica \\ *Autor para contacto: cesarlaurito@ice.co.cr
}

(Recibido: 26/07/2011; aceptado: 28/11/2011)

\begin{abstract}
Central America Proboscidea Gomphotherium hondurensis (Frick, 1933), recovered at the Valley of Torola River is described here. This locality corresponds with the outcrops of the Corinto local fauna, previously studied and defined by the American paleontologists David S. Webb and Stephen Perrigo during the 70's years; which is considered as one of the most important fossiliferous localities of the Upper Miocene of Central America.

Keywords: Gomphoterioidea, Upper Miocene, Morazán Formation, El Salvador.

Resumen: se describe un molar inferior, aislado, del proboscídeo endémico de América Central Gomphotherium hondurensis (Frick, 1933), recuperado en el valle del río Torola en la localidad fosilífera de Corinto, la cual fue estudiada y caracterizada en los años 70 por los paleontólogos estadounidenses David S. Webb y Stephen Perrigo; la fauna local de Corinto es considerada una de las más importantes localidades fosilíferas del Mioceno Superior de América Central. Palabras clave: Gomphoterioidea, Mioceno Superior, Formación Morazán, El Salvador.
\end{abstract}




\section{INTRODUCCIÓN}

En América Central septentrional se han registrado 3 familias de Proboscídeos con 4 géneros, Elephantidae con el género Mammuthus (Laurito \& Aguilar, 2006); Mammutidae con el género Mammut (Lucas \& Alvarado, 1991) y Gomphotheriidae con los géneros Gomphotherium y Cuvieronious (Lucas \& Alvarado, 2010). De estas familias, la Gomphotheriidae es la que se le atribuyen los registros más antiguos del área, haciendo su aparición en el Mioceno Medio de la Formación Gracias en Honduras, con el género Gomphotherium (Frick, 1933), y extendiéndose su rango hasta el Pleistoceno tardío con el género Cuvieronius frecuente en diversas localidades de Guatemala, Honduras, El Salvador y el resto de América Central (Leidy, 1859; Laurito, 1988; Montellano-Ballesteros, 2002; Pearson, 2005; Cisneros, 2005; Jackson \& Fernández, 2005).

Por otro lado, el género Gomphotherium en América Central está representado por una única especie Gomphotherium hondurensis (Frick, 1933), anteriormente incluida dentro del género Rhynchotherium y la especie Rhynchotherium blicki. Debido a ello, el género Rhynchotherium fue considerado endémico de América del Norte y/o América Central sensu Lambert, 1996; Webb \& Perrigo, 1984. A su vez el género Gomphotherium cuenta con un amplio registro en el Cenozoico tardío de Norte América, incluyendo México (Ferrusquía-Villafranca, 1990, Miller \& Carranza-Castañeda, 2001, Arroyo-Cabrales et al., 2007). Sin embargo, América Central registra una única especie endémica (Webb \& Perrigo, 1984), G. hondurensis (Frick, 1933) conocida por un abundante registro fósil en Honduras y El Salvador (Frick, 1933; Webb \& Perrigo, 1984; Miller \& Carranza-Castañeda, 2001) y por individuos aislados en Guatemala (Lucas \& Alvarado, 1995) y Costa Rica (Laurito \& Valerio, 2007; Valerio \& Laurito, 2008).

El material del presente estudio fue recuperado en setiembre del 2009, procede de la localidad de Corinto ubicada a $4 \mathrm{~km}$ al $\mathrm{N} 73^{\circ} \mathrm{W}$ del pueblo del mismo nombre en el Valle del Río Torola, Cantón de Calavera, Municipio de Cacaopera,
Departamento de Morazán, en las coordenadas N $13^{\circ} 49^{\prime} 02.2^{\prime \prime}$ y W 88 00'22.7' (Fig. 1).

\section{GEOLOGÍA}

De acuerdo con el mapa geológico de la república de El Salvador, publicado por el Ministerio de Medio Ambiente y Recursos Naturales (2002). la Formación Morazán está constituida por 4 miembros todos de origen volcánico. La localidad donde se recolectó el espécimen corresponde con los miembros medios caracterizados por piroclastitas intermedias hasta intermedias-ácidas, epiclastitas volcánicas, efusivas y efusivas intermedias hasta intermedias-ácidas, piroclastitas y alteración regional por influencia hidrotermal.

El problema con la definición de formaciones geológicas en El Salvador, es que muchos de los sedimentos aluviales, fluviales, lacustres o incluso marinos son descritos en función de los componentes líticos de los clastos sedimentarios, mayoritariamente volcanogénicos y no de acuerdo a los ambientes sedimentarios en los que se depositaron, a excepción de los sedimentos aluviales o fluviales cuaternarios o arrecifales del Cretácico Superior. Ejemplo de ello son las descripciones de localidades altamente fosilíferas, donde se indica que "los fósiles están embebidos en un flujo conglomerádico de composición andesítica" cf. Stirton \& Gealey $(1943,1949)$ o la localidad fosilífera de Tomayate, cuyo basamento está constituido por arcillas azul verdosas con abundantes foraminíferos, sin embargo están cartografiadas como de origen volcaniclástico a pesar de ser marinas. Igual sucede con las secuencias superiores de esta localidad, altamente fosilíferas y consideradas epiclastitas, pero en realidad son sedimentos de origen fluvial (Laurito observ. personal).

El molar recuperado se encontró en sedimentos de origen aluvial clasificados como piroclastitas que de acuerdo a Webb \& Perrigo (1984), irrumpen entre una secuencia inferior y superior de ignimbritas que corresponderían con las ignimbritas Mio-Pliocénicas descritas por Williams \& McBirney (1969) en áreas adyacentes de Honduras y que en El Salvador se 

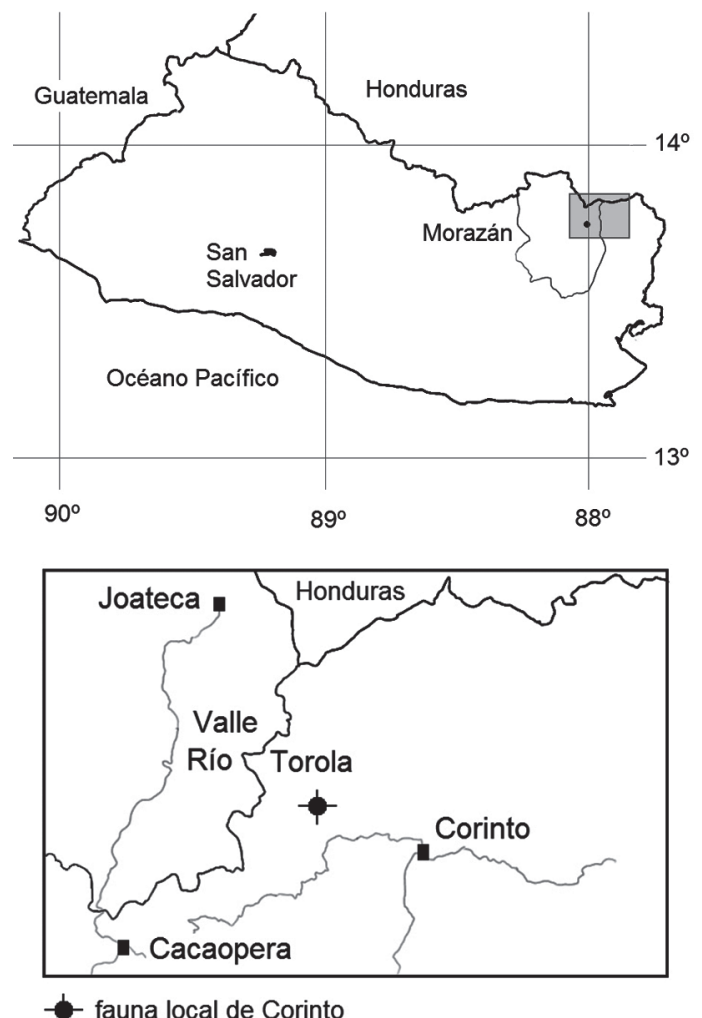

Fig. 1: Mapa de ubicación de la fauna local de Corinto, Departamento de Morazán, Cantón de Calavera, República de El Salvador

les incluye en la Formación Morazán de edad Oligoceno? - Mioceno. Webb \& Perrigo (1984). Además, describen estos depósitos aluviales como intercalaciones de areniscas y arcillas junto con ocasionales lentes de conglomerados constituidos por grabas con clastos de pómez de hasta $40 \mathrm{~mm}$ de diámetro, en algunas zonas estos sedimentos se encuentran intercalados con capas de toba y estratos de areniscas y pueden alcanzar espesores totales de $10 \mathrm{~m}$.

\section{PALEONTOLOGÍA}

Orden PROBOSCIDEA (Illiger, 1811)

Superfamilia GOMPHOTHERIODEA, Hay 1922 Familia GOMPHOTERIIDAE (Cabrera, 1929) Género GOMPHOTHERIUM Burmeister, 1837
Especie Gomphotherium hondurensis (Frick, 1933) Lucas \& Morgan, 2008

Material: tercer molar inferior derecho [m3], depositado en la Casa de la Cultura de Cacaopera, Departamento de Morazán bajo el código 4m CCCA- 01 (Fig. 2).

Descripción: molar relativamente pequeño, con una longitud antero-posterior de $128 \mathrm{~mm}$ con corona baja, elongada y un estado intermedio de desgaste. La corona presenta cuatro lófidos y un cíngulo reducido observable en la zona lingual del primer cónulo postrito; los tres lófidos anteriores muestran un desgaste moderado, con longitudes linguo-vestibulares de $49 \mathrm{~mm}, 55$ $\mathrm{mm}$ y $52 \mathrm{~mm}$ respectivamente; el cuarto lófido presenta un desgaste incipiente, más un talónido con dos cónulos reducidos, todos separados por un sulcus mediano.

El primer hemilófido pretrito se encuentra muy desgastado y muestra una figura en forma de trébol muy amplio y sesgado, en tanto el hemilófido postrito es de contorno groseramente rectangular; el segundo hemilófido pretrito presenta un contorno en forma de trébol simétrico, al igual que el tercero aunque este último es notablemente más pequeño comparado con el primero y segundo hemilófidos pretritos; el hemilófido postrito segundo presenta contorno triangular y su ancho es similar al del primer hemilófido, en tanto que el tercer hemilófido postrito es notablemente rectangular y angosto.

La raíz presenta dos ramos radiculares fusionados correspondientes al primer lófido y 5 ramos radiculares fusionados correspondientes a los lófidos segundo, tercero y cuarto.

Discusión: el molar en cuestión se distingue de los similares del género Cuvieronius por su relativo pequeño tamaño, a pesar de que se trata de un molar tercero inferior que corresponde al más grande de la serie dental; sus dimensiones nos indican que se trata de un Gomphotheriidae de muy pequeña talla y el patrón de desgaste de los cónulos, muy simples, sugiere que se trata de una forma relativamente primitiva, característica de los mastodontes del Mioceno Medio y Superior; por 


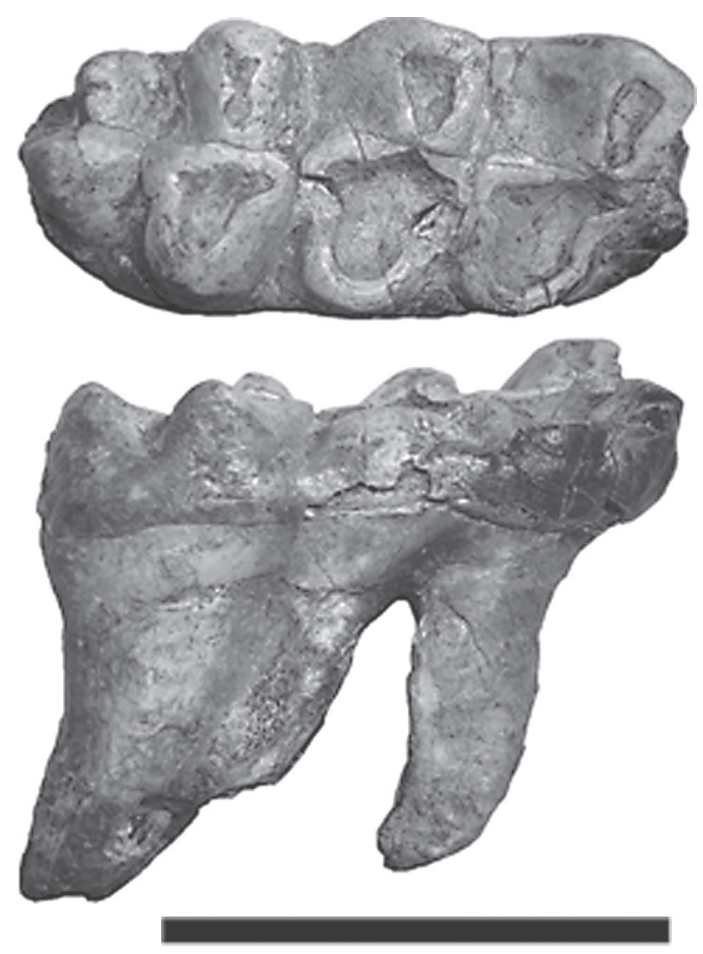

Fig. 2: tercer molar inferior derecho de Gophotherium hondurensis (Frick, 1933), imagen superior en norma oclusal; imagen inferior en vista labial. Escala gráfica $10 \mathrm{~cm}$

ejemplo el hemilófido postrito segundo de contorno triangular con la base hacia la región lingual, es muy similar al molar ilustrado por Frick (1933) en la figura 5 o por Webb \& Perrigo (1984) en la figura 4. De igual forma, su longitud anteroposterior es ligeramente más pequeña que el rango inicialmente descrito por Frick (1933) y Webb \& Perrigo (1984) para los m3 que varía entre los $139 \mathrm{~mm}$ (rango menor) para la forma Aybelodon hondurensis y los $185 \mathrm{~mm}$ (rango mayor) para la forma Rhynchotherium blicki; pero por estar conformado por 4 lófidos y 2 cónulos no queda duda de que se trata de un tercer molar y su patrón no es diferenciable del observado para la especie Gomphotherium hondurensis (Frick, 1933); la única diferencia es su tamaño ligeramente menor, pero podría deberse a que el ejemplar correspondería a una hembra y por lo tanto sería evidencia de dimorfismo sexual en la especie $G$. hondurensis (Frick, 1933), sobre todo porque el ancho de los lófidos cae en el rango observado de $\operatorname{los} 3 \mathrm{~m} 3$.

\section{Distribución paleogeográfica, paleoecología y edad}

La especie Gomphotherium hondurensis (Frick, 1933) ha sido registrada en el Mioceno Medio de la Formación Gracias en Honduras (Webb \& Perrigo, 1984), Mioceno Superior de El Salvador en la localidad fosilífera de Corinto (Webb \& Perrigo, 1984); Guatemala en la Formación Herrería (Lucas \& Alvarado, 1995) y Costa Rica en las localidades del Hemphilliano tardío- temprano de Santa Rita y Limoncito, Formación Curré (Laurito \& Valerio, 2005; Valerio \& Laurito, 2008; Valerio, 2010).

Desde el punto de vista paleoecológico la especie G. hondurensis habitó, según la interpretación de Webb \& Perrigo (1984) en un ambiente de sabanas arboladas y humedales para la formación Gracias en Honduras y Corinto en El Salvador, y Valerio (2010) asume un ambiente de tierras bajas con desarrollo de sabanas arboladas y humedales costeros para la fauna asociada a la especie Gomphotherium hondurensis (Frick, 1933) en Costa Rica.

Aunque el molar estudiado fue recuperado en unidades "epiclásticas" de los miembros medios de la Formación Morazán, está claro que proviene de sedimentos aluviales correlacionables con la localidad fosilífera de Corinto o fauna local de Corinto sensu Webb \& Perrigo (1984), ubicada entre dos unidades ignimbríticas y datada por dichos autores como Hemphilliano temprano fundamentada en el registro de Osteoborus cynoides, Hippotherium plicatile y Calippus hondurensis.

\section{CONCLUSIONES}

Se describe un nuevo registro del proboscídeo endémico de América Central Gomphotherium hondurensis (Frick, 1933) para la localidad fosilífera de Corinto de edad Mioceno Superior o 
Hemphilliano temprano, Formación Morazán; esta fauna se correlaciona con los registros fósiles de la Formación Gracias en Honduras.

\section{AGRADECIMIENTO}

Queremos hacer patente nuestro agradecimiento a la Sra. María Luisa Fuentes de Claro, Directora de la Casa de la Cultura de Cacaopera, por permitirnos el espécimen, objeto de estudio en este trabajo.

\section{BIBLIOGRAFÍA}

ANÓNIMO, 2002: Mapa Geológico de El Salvador.- Escala 1:250 000. Ibérica de Estudios e Ingeniería S.A., Ministerio de Medio Ambiente y Recursos Naturales.

ARROYO-CABRALES, J., POLACO, O.J., LAURITO, C.A., JOHNSON, E., ALBERDI, M.T. \& VALERIO A.L., 2007: The Proboscideans (Mammalia) from Mesoamerica.- En AGENBROAD, L., HAYNES, G., JOHNSON, E. \& PALOMBO, M.R. (eds.): World of Elephants 2: Selected papers from the $2^{\text {nd }}$ Congress, Mammoth Site of Hot Springs.- Quat. Int. 169-170: 17-23.

CISNEROS, J.C., 2005: New Pleistocene vertebrate fauna from El Salvador.- Rev. Bras. Paleontol. 8(3): 239-255.

FERRUSQUÍA-VILLAFRANCA， I., 1990: Biostratigraphy of the Mexican continental Miocene: part II, the southeastern (Oaxacan) faunas.- Paleont. Mexicana, Inst. de Geol., Univ. Nac. Autónoma de México, 56: 57-109.

FRICK, C., 1933: New remains in trilopho-donttetrabelodont mastodonts.- Bull. Amer. Mus. Nat. Hist. 59: 505-652.
JACKSON, D.R. \& FERNANDEZ, E., 2005: A small Pleistocene Mammalia megafauna from southern Honduras.- En: HULBERT, R.C., MORGAN, G.S. \& BASKIN, J.A. (eds.): Cenozoic vertebrates of the Americas: Papers to Honor S. David Webb.- Bull. Fla. Mus. Nat. Hist. 45(4): 267-276

LAMBERT, W.D., 1996: The biogeography of the gomphotheriid proboscideans of North America.- In: SHOSHANI, J., TASSY, P. (eds.), The Proboscidea. Evolution and palaeoecology of elephants and their relatives.- Oxford Univ. Press, Oxford: 143-148.

LAURITO, C.A., 1988: Los Proboscídios fósisles de Costa Rica y su contexto en la América Central.- Vínculos, 14: 29-58.

LAURITO, C.A. \& AGUILAR, D.H., 2006: El registro de Mammuthus (Proboscidea, Elephantidae) en la República de El Salvador, América Central.- Rev. Geol. Amér. Central, 34-35: 73-81.

LAURITO, C.A. \& VALERIO, A.L., 2007: First record of Rhynchotherium blicki (Frick, 1933) for the late Cenozoic of Costa Rica.Rev. Geol. Amér. Central, 33: 75-82.

LEIDY, J., 1859: On mastodon tooth from Honduras.- Proc. Acad. Nat. Sci. of Philadelphia, 2: 91.

LUCAS, S.G. \& ALVARADO, G.E., 1991: El hallazgo más austral de Mammut americanum: el caso del mastodonte de San Pedro Sula, Honduras.- Rev. Geol. Amér. Central, 13: 85-89.

LUCAS, S.G. \& ALVARADO, G.E., 1995: El proboscídeo Rhynchotherium blicki (Mioceno Tardío) del oriente de Guatemala.- Rev. Geol. Amér. Central, 18: 19-24. 
LUCAS, S.G. \& MORGAN, G.S., 2008: Taxonomy of Rhynchotherium (Mammalia, Proboscidea) from the Miocene-Pliocene of North America.- New Mex. Mus. Nat. Hist. Sci. Bull. 44: 71-87.

MILLER, W.E. \& CARRANZA-CASTAÑEDA, O., 2001: Late Cenozoic mammals from the basins of central México.- Bull. Italian Paleont. Soc. 40: 235-242.

MONTELLANO-BALLESTEROS, M., 2002: New Cuvieronius finds from the Pleistocene of Central México.- J. Paleont. 76: 578-583.

PEARSON, G.A., 2005: Late Pleistocene megafaunal deposits on the Isthmus of Panama and their paleoenvironmental implications.- Caribbean J. Sc. 41(1): 1-13.

MILLER, W.E. \& CARRANZA-CASTAÑEDA, O., 2001: Late Cenozoic mammals from the basins of central México.- Bull. Italian Paleont. Soc. 40: 235-242.

STIRTON, R.A. \& GEALEY, W.K. 1943: Fossil vertebrates from El Salvador.- J. Paleont. 17(3): 309.
STIRTON, R.A. \& GEALEY, W.K. 1949: Reconnaissance geology and vertebrate paleontology of El Salvador, Central America.- Bull. Geol. Soc. Amer. 60:17311764.

VALERIO, A.L., 2010: Paleontología, bioestratigrafía y paleoecología de los caballos fósiles de la Formación Curré en el Cantón de Coto Brus, Costa Rica (análisis basado en material dental).- 353 págs. Univ. de Costa Rica, San José [Tesis Lic.].

VALERIO, A.L. \& LAURITO, C.A., 2008: Dental remains of inmature individuals of Rhynchotherium blicki (Frick, 1933) of San Gerardo de Limoncito, Coto Brus, Costa Rica.- Rev. Geol. Amér. Central, 39: 87-91.

WEBB, D.S. \& PERRIGO, S.C., 1984: Late Cenozoic vertebrates from Honduras and El Salvador.- En: MACFADDEN, B.J. (ed.): Origin and evolution of the Cenozoic vertebrate fauna of Middle America.- J. Vert. Paleont. 4(2):237-254. 